\title{
Hypersphere World-Universe Model: Centre of Our Galaxy
}

\author{
Vladimir S. Netchitailo \\ Biolase Inc., Irvine, USA \\ Email: netchitailov@gmail.com
}

How to cite this paper: Netchitailo, V.S. (2022) Hypersphere World-Universe Model: Centre of Our Galaxy. Journal of High Energy Physics, Gravitation and Cosmolo$g y, 8,25-55$.

https://doi.org/10.4236/jhepgc.2022.81003

Received: October 15, 2021

Accepted: November 20, 2021

Published: November 23, 2021

Copyright $\odot 2022$ by author(s) and Scientific Research Publishing Inc. This work is licensed under the Creative Commons Attribution International License (CC BY 4.0).

http://creativecommons.org/licenses/by/4.0/ (c) (i) Open Access

\begin{abstract}
In 1937, Paul Dirac proposed: the Large Number Hypothesis and the Hypothesis of the variable gravitational "constant"; and later added the notion of continuous creation of Matter in the World. The developed Hypersphere World-Universe Model (WUM) follows these ideas, albeit introducing a different mechanism of matter creation. In this paper, we show that WUM is a natural continuation of Classical Physics, and it can already serve as a basis for a New Cosmology proposed by Paul Dirac. In 2013, WUM predicted the values of the following Cosmological parameters: gravitational, concentration of intergalactic plasma, and the minimum energy of photons, which were experimentally confirmed in 2015-2018. "The Discovery of a Supermassive Compact Object at the Centre of Our Galaxy" (Nobel Prize in Physics 2020) made by Prof. R. Genzel and A. Ghez is a confirmation of one of the most important predictions of WUM in 2013: "Macroobjects of the World have cores made up of the discussed DM particles. Other particles, including DM and baryonic matter, form shells surrounding the cores".
\end{abstract}

\section{Keywords}

Law of Conservation of Angular Momentum, Dark Epoch, Rotational Fission, Luminous Epoch, Multicomponent Dark Matter, Milky Way Centre, Medium of the World, Dark Matter Reactors

\section{Introduction}

In our view, we should make use of a number of hypotheses unknown and forgotten by mainstream scientific community in order to elaborate a New Cosmology. Below we will describe the Hypotheses belonging to classical physicists such as Newton, Le Sage, McCullagh, Riemann, Heaviside, Tesla, and Dirac and develop them in frames of WUM. Please pay tribute to these great physicists! 
The presented Hypotheses are not new, and we do not claim credit for them. In fact, we are developing the existent Hypothesis and proposing new Hypothesis in frames of WUM. The main objective of the Model is to unify and simplify existing results in Classical Physics into a single coherent picture of a New Cosmology.

Many results obtained in WUM are quoted in the current work without a full justification; an interested reader is encouraged to view the referenced papers in such cases.

Cosmology is a branch of Classical Physics. It should then be described by classical notions, which define emergent phenomena. By definition, an emergent phenomenon is a property that is a result of simple interactions that work cooperatively to create a more complex interaction. Physically, simple interactions occur at a microscopic level, and the collective result can be observed at a macroscopic level.

\section{Classical Physics}

In this Section we describe principal milestones in Classical Physics. Based on the analysis of measured physical constants we conclude that the most important Fundamental constants could be calculated before Quantum Mechanics [1].

Maxwell's equations were published by J. C. Maxwell in 1861 [2]. He calculated the velocity of electromagnetic waves from the value of the electrodynamic constant $c$ measured by Weber and Kohlrausch in 1857 [3] and noticed that the calculated velocity was very close to the velocity of light measured by Fizeau in 1849 [4]. This observation made him suggest that light is an electromagnetic phenomenon [5].

We emphasize that $c$ in Maxwell's equations is the electrodynamic constant but not the speed of light in vacuum. By definition, the electrodynamic constant $c$ is the ratio of the absolute electromagnetic unit of charge $e$ to the absolute electrostatic unit of charge $e / c$, where $e$ is the elementary charge. It is worth noting that the speed of light in vacuum, commonly denoted as $c$, is not related to the World in our Model, because there is no Vacuum in It. Instead, there is the Medium of the World consisting of elementary particles.

Rydberg constant $R_{\infty}$ is a physical constant relating to atomic spectra. The constant first arose in 1888 as an empirical fitting parameter in the Rydberg formula for the hydrogen spectral series [6].

Electron Charge-to-Mass Ratio $e / m_{e}$ is a Quantity in experimental physics. It bears significance because the electron mass $m_{e}$ cannot be measured directly. The $e / m_{e}$ ratio of an electron was successfully measured by J. J. Thomson in 1897 [7]. We name it after Thomson: $R_{T} \equiv e / m_{e}$.

Planck Constant $h$ was suggested by Max Planck in 1901 as the result of investigating the problem of black-body radiation. He used Boltzmann's equation from Statistical Thermodynamics: $S=k_{B} \ln W$ that shows the relationship between entropy $S$ and the number of ways the atoms or molecules of a thermo- 
dynamic system can be arranged ( $k_{B}$ is the Boltzmann constant) [8].

Based on the experimentally measured values of the constants $R_{\infty}, R_{T}, \mathcal{C}, h$ we calculate the most important constants in WUM as follows [1]:

- Basic unit of size a:

$$
a=0.5\left[8\left(\mu_{0} h / c\right)^{3} R_{\infty} R_{T}^{6}\right]^{1 / 5}=1.7705641 \times 10^{-14} \mathrm{~m}
$$

- Dimensionless Rydberg constant $\alpha$ :

$$
\alpha=\left(2 a R_{\infty}\right)^{1 / 3}
$$

where $\mu_{0}$ is a magnetic constant (or vacuum permeability):

$\mu_{0}=4 \pi \times 10^{-7} \mathrm{H} / \mathrm{m}$. It is worth noting that the constant $\alpha$ was later named "Sommerfeld's constant" and subsequently "Fine-structure constant".

The calculated value of $\alpha^{-1}$ is:

$$
\alpha^{-1}=137.0359991
$$

WUM is based on two parameters only: dimensionless Rydberg constant $\alpha$ and time-varying Quantity $Q$ that is a measure of the Size $R$ and Age $A_{\tau}$ of the World and is, in fact, the Dirac Large Number $\left(t_{0}\right.$ is a basic unit of time: $\left.t_{0}=a / c\right)$ :

$$
Q=\frac{R}{a}=\frac{A_{\tau}}{t_{0}}
$$

which in present epoch equals to: $Q=0.759972 \times 10^{40}$.

\section{Hypotheses Revisited by WUM}

\subsection{Aether}

Physical Aether was suggested as early as 17th century, by Isaac Newton. Following the work of Thomas Young (1804) and Augustin-Jean Fresnel (1816), it was believed that light propagates as a transverse wave within an elastic medium called Luminiferous Aether. At that time, it was realized that Aether could not be an elastic matter of an ordinary type that can only transmit longitudinal waves.

Unique properties of Aether were discussed by James McCullagh in 1846 who proposed a theory of a rotationally elastic medium, i.e., a medium in which every particle resists absolute rotation. This theory produces equations analogous to Maxwell's electromagnetic equations [9]. Aether with these properties can transmit transverse waves. Luminiferous Aether was abandoned in 1905.

In later years there have been classical physicists who advocated the existence of Aether:

- Nikola Tesla declared in 1937 in "Prepared Statement on the 81st birthday observance": "All attempts to explain the workings of the universe without recognizing the existence of the aether and the indispensable function it plays in the phenomena are futile and destined to oblivion" [10];

- Paul Dirac stated in 1951 in the article in Nature, titled "Is there an Aether?" 
that "we are rather forced to have an aether" [11].

There are no Luminiferous Aether and Vacuum in WUM. The Model introduces the Medium of the World, which is composed of stable elementary particles: protons, electrons, photons, neutrinos, and Dark Matter Particles (DMPs). The existence of the Medium is a principal point of WUM. It follows from the observations of Intergalactic Plasma; Cosmic Microwave Background Radiation; Far-Infrared Background Radiation. According to WUM, inter-galactic voids discussed by astronomers are, in fact, examples of the Medium in its purest. The Medium is the absolute frame of reference.

\subsection{Le Sage's Theory of Gravitation}

Wikipedia summarizes this theory as "a mechanical explanation for Newton's gravitational force in terms of streams of tiny unseen particles (which Le Sage called ultra-mundane corpuscles) impacting all material objects from all directions. According to this model, any two material bodies partially shield each other from the impinging corpuscles, resulting in a net imbalance in the pressure exerted by the impact of corpuscles on the bodies, tending to drive the bodies together".

According to WUM, the energy density of the Medium $\rho_{M}$ is $2 / 3$ of the total energy density of the World $\rho_{W}$ in all cosmological times. The energy density of all Macroobjects adds up to $1 / 3$ of $\rho_{W}$ throughout the World's evolution. The relative energy density of DMPs is about $92.8 \%$ and Ordinary Particles (protons, electrons, photons, and neutrinos)—about 7.2\%. A time-varying gravitational parameter $G$ is proportional to the time-varying $\rho_{M}$ [12]. In frames of WUM:

- DMPs are "Le Sage’s ultra-mundane corpuscles";

- Le Sage's theory of gravitation defines Gravity as an emergent phenomenon;

- Gravity is not an interaction but a manifestation of the Medium.

\subsection{Hypersphere Universe}

In 1854, Georg Riemann proposed Hypersphere as a model of a finite universe [13].

WUM: Before the Beginning of the World there was nothing but an Eternal Universe. About 14.22 billion years ago the World was started by a fluctuation in the Eternal Universe, and the Nucleus of the World, a 4D ball, was born. An extrapolated Nucleus radius at the Beginning was equal to $a$. The Finite World is a $3 \mathrm{D}$ Hypersphere that is the surface of the $4 \mathrm{D}$ Nucleus. All points of the hypersphere are equivalent; there are no preferred centers or boundary of the World [14]. The extrapolated energy density of the World at the Beginning was four orders of magnitude smaller than the nuclear energy density [15].

\subsection{Gravitoelectromagnetism}

Gravitoelectromagnetism (GEM) refers to a set of formal analogies between the 
equations for Electromagnetism and relativistic gravitation. GEM is an approximation to Einstein's field equations for General Relativity in the weak field limit. H. Thirring pointed out this analogy in his "On the formal analogy between the basic electromagnetic equations and Einstein's gravity equations in first approximation" paper published in 1918 [16]. The equations for GEM were first published in 1893 by O. Heaviside as a separate theory expanding Newton's law [17].

WUM follows this theory. In most cases of weak gravitational fields, we can neglect the influence of General Relativity effects. For example, the surface gravity of the Earth equals: $g=9.80665 \mathrm{~m} \cdot \mathrm{s}^{-2}$ and a general relativity acceleration is $\sim 3 \times 10^{-10} \mathrm{~m} \cdot \mathrm{s}^{-2}$ [18]. In case of strong gravitational fields, we should use the Einstein's field equations for General Relativity.

\subsection{Dirac Large Number Hypothesis}

In 1937, Paul Dirac in the paper "A new basis for cosmology" said [19]:

"Since general relativity explains so well local gravitational phenomena, we should expect it to have some applicability to the universe as a whole. We cannot, however, expect it to apply with respect to the metric provided by the atomic constants, since with this metric the 'gravitational constant' is not constant but varies with the epoch. We have, in fact, the ratio of the gravitational force to the electric force between electron and proton varying in inverse proportion to the epoch, and since, with our atomic units of time, distance and mass, the electric force between electron and proton at a constant distance apart is constant, the gravitational force between them must be inversely proportional to the epoch. Thus, the gravitational constant will be inversely proportional to the epoch".

In Summary, he concluded:

"It is proposed that all the very large dimensionless numbers which can be constructed from the important natural constants of cosmology and atomic theory are connected by simple mathematical relations involving coefficients of the order of magnitude unity. The main consequences of this assumption are investigated, and it is found that a satisfactory theory of cosmology can be built up from it'.

WUM follows an idea of time-varying $G$ and introduces a dimensionless time-varying quantity $Q$, that is, in fact, the Dirac Large Number, which in present epoch equals to: $Q=0.759972 \times 10^{40}$. $G$ can be calculated from the value of the parameter $Q[14]$ :

$$
G=\frac{a^{2} c^{4}}{8 \pi h c} \times Q^{-1}=\frac{a^{3} c^{3}}{8 \pi h c} \times \tau^{-1}
$$

WUM holds that there indeed exist simple mathematical relations between all Primary Cosmological Parameters (PCPs) that depend on $Q$ (see Section 4.1.):

- Concentration of Intergalactic Plasma $n_{I G P}$; 
- Minimum Energy of Photons $E_{p h}$;

- Temperature of the Microwave Background Radiation $T_{M B R}$;

- Temperature of the Far-Infrared Background Radiation peak $T_{\text {FIRB }}$.

These PCPs belong to the Medium of the World. There are no Aether and Vacuum in WUM.

\subsection{Creation of Matter}

In 1964, F. Hoyle and J. V. Narlikar offered an explanation for the appearance of new matter by postulating the existence of what they dubbed the "Creation field" [20].

In 1974, Paul Dirac discussed continuous creation of matter by additive (uniformly throughout space) and multiplicative mechanism (proportional to the amount of existing matter) [21].

WUM: The 3D World, which is a Hypersphere of 4D Nucleus, was started by a fluctuation in the Eternal Universe. 4D Nucleus is expanding in Its fourth spatial dimension, and Its surface, the Hypersphere, is likewise expanding. The radius of the Nucleus $R$ is increasing with speed $c$ (gravitodynamic constant) for the absolute cosmological time $\tau$ from the Beginning and equals to $R=c \tau$. By definition, the gravitodynamic constant $c$ is the ratio of the absolute gravitomagnetic unit of charge $E_{0}$ to the absolute gravitostatic unit of charge $E_{0} / c$, where $E_{0}$ is a basic unit of energy: $E_{0}=h c / a$.

The surface of the Nucleus is created in a process analogous to sublimation. Continuous creation of matter is the result of this process. Sublimation is a wellknown endothermic process that happens when surfaces are intrinsically more energetically favorable than the bulk of a material, and hence there is a driving force for surfaces to be created.

Dark Matter (DM) is created by the Universe in the 4D Nucleus of the World. Dark Matter Particles (DMPs) carry new DM into the 3D Hypersphere World. Ordinary Matter is a byproduct of DMPs self-annihilation. Consequently, a Matter-Antimatter Asymmetry problem discussed in literature does not arise (since antimatter does not get created by DMPs self-annihilation). By analogy with 3D ball, which has two-dimensional sphere surface (that has surface energy), we can imagine that the 3D Hypersphere World has a "Surface Energy" of the 4D Nucleus.

The proposed process is a $4 \mathrm{D}$ process responsible for the expansion, creation of Matter and arrow of Time. It is a Hypothesis of WUM. In our view, the arrow of the Cosmological Time does not depend on any physical phenomenon in the Medium of the World. It is the result of the Worlds' expansion due to a driving force for surfaces to be created.

It is important to emphasize that:

- Creation of Matter is a direct consequence of expansion;

- Creation of DM occurs homogeneously in all points of the Hypersphere World. 


\subsection{Multi-Component Dark Matter}

Two-component DM system consisting of bosonic and fermionic components is proposed for the explanation of emission lines from the bulge of Milky Way galaxy. C. Boehm, P. Fayet, and J. Silk propose a way "to reconcile the low and high energy signatures in gamma-ray spectra, even if both of them turn out to be due to Dark Matter annihilations. One would be a heavy fermion for example, like the lightest neutralino (>100 GeV), and the other one a possibly light spin-O particle ( 100 MeV). Both of them would be neutral and also stable" [22].

WUM proposes multicomponent DM system consisting of two couples of coannihilating DMPs: a heavy Dark Matter Fermion (DMF)—DMF1 (1.3 TeV) and a light spin-0 boson-DIRAC $(70 \mathrm{MeV})$ that is a dipole of Dirac's monopoles with charge $\mu=e / 2 \alpha$; a heavy fermion-DMF2 $(9.6 \mathrm{GeV})$ and a light spin-0 boson-ELOP $(340 \mathrm{keV})$ that is a dipole of preons with electrical charge $e / 3$; a self-annihilating fermion-DMF3 $(3.7 \mathrm{keV})$ and a fermion DMF4 $(0.2 \mathrm{eV})$.

WUM postulates that rest energies of DMFs and bosons are proportional to the basic unit of energy $E_{0}$ multiplied by different exponents of $\alpha$ and can be expressed with the following formulae:

DMF1 (fermion): $E_{D M F 1}=\alpha^{-2} E_{0}=1.3149950 \mathrm{TeV}$,

DMF2 (fermion): $E_{D M F 2}=\alpha^{-1} E_{0}=9.5959823 \mathrm{GeV}$,

DIRAC (boson): $E_{\text {DIRAC }}=\alpha^{0} E_{0}=70.025267 \mathrm{MeV}$,

ELOP (boson): $E_{E L O P}=2 / 3 \alpha^{1} E_{0}=340.66606 \mathrm{keV}$,

DMF3 (fermion): $E_{D M F 3}=\alpha^{2} E_{0}=3.7289402 \mathrm{keV}$,

DMF4 (fermion): $E_{D M F 4}=\alpha^{4} E_{0}=0.19857111 \mathrm{eV}$.

It is worth noting that the rest energy of electron $E_{e}$ equals to: $E_{e}=\alpha E_{0}$ and the Rydberg unit of energy is: $R y=h c R_{\infty}=0.5 \alpha^{3} E_{0}=13.605693 \mathrm{eV}$.

We still do not have a direct confirmation of DMPs' rest energies, but we do have a number of indirect observations. The signatures of DMPs self-annihilation with expected rest energies of $1.3 \mathrm{TeV}$; $9.6 \mathrm{GeV} ; 70 \mathrm{MeV} ; 340 \mathrm{keV} ; 3.7 \mathrm{keV}$ are found in spectra of the diffuse gamma-ray background and the emission of various Macroobjects in the World. We connect observed gamma-ray spectra with the structure of Macroobjects (nuclei and shells composition). Self-annihilation of those DMPs can give rise to any combination of gamma-ray lines. Thus, the diversity of Very High Energy gamma-ray sources in the World has a clear explanation in WUM [15].

In this regard, it is worth recalling a story about neutrinos: "The neutrino was postulated first by W. Pauli in 1930 to explain how beta decay could conserve energy, momentum, and angular momentum (spin). But we still don't know the values of neutrino masses". Although we still cannot measure neutrinos' masses directly, no one doubts their existence.

\subsection{Macroobjects}

The existence of supermassive objects in galactic centers is now commonly accepted. Many non-traditional models explaining supermassive dark objects ob- 
served in galaxies and galaxy clusters are widely discussed in literature [23]-[29]. The prospect that DMPs might be observed in Centers of Macroobjects has drawn many new researchers to the field [30] [31]. Indirect effects in cosmic rays and gamma-ray background from the annihilation of DM in the form of heavy stable neutral leptons in Galaxies were considered in pioneer articles [32]-[37].

Observational data like dynamics of galaxies and star formation disfavor exotic cold and warm DM proposed in the Standard Cosmology. In his famous paper [38], Prof. P. Kroupa stated that "all observational quantities that are derived at present, such as star-formation rate densities, distances and ages from redshifts, and galaxy masses, are likely to require possibly major revision".

According to WUM, Macrostructures of the World (Superclusters, Galaxies, Extrasolar systems) have Nuclei made up of DMFs, which are surrounded by Shells composed of DM and baryonic matter. The shells envelope one another, like a Russian doll. The lighter a particle, the greater the radius and the mass of its shell. Innermost shells are the smallest and are made up of heaviest particles; outer shells are larger and consist of lighter particles [39].

Table 1 describes the parameters of Macroobjects Cores (which are Fermionic Compact Stars in WUM) in the present Epoch made up of different DM fermions: self-annihilating DMF1, DMF2, DMF3, fermion DMF4, and Electron-Positron plasma.

The calculated parameters of the shells show that [39]:

- Nuclei made up of DMF1 and/or DMF2 compose Cores of stars in extrasolar systems;

- Shells of DMF3 and/or Electron-Positron plasma around Nuclei made up of DMF1 and/or DMF2 make up Cores of galaxies;

- Nuclei made up of DMF1 and/or DMF2 surrounded by shells of DMF3 and DMF4 compose Cores of superclusters.

Macroobjects' Cores have the following properties:

- The minimum radius of Core $R_{\min }$ made up of any fermion equals to three Schwarzschild radii;

- Core density does not depend on $M_{\max }$ and $R_{\min }$ and does not change in time while $M_{\max } \propto \tau^{3 / 2}$ and $R_{\min } \propto \tau^{1 / 2}$;

Table 1. Parameters of Macroobjects cores made up of different fermions in present Epoch.

\begin{tabular}{ccccc}
\hline Fermion & $\begin{array}{c}\text { Fermion } \\
\text { Mass } \boldsymbol{m}_{\mathfrak{f}} \\
\mathrm{MeV}\end{array}$ & $\begin{array}{c}\text { Macroobject } \\
\text { Mass } \\
\boldsymbol{M}_{\max }, \mathrm{kg}\end{array}$ & $\begin{array}{c}\text { Macroobject } \\
\text { Radius } \\
\boldsymbol{R}_{\min }, \mathrm{m}\end{array}$ & $\begin{array}{c}\text { Macroobject } \\
\text { Density } \\
\boldsymbol{\rho}_{\text {max }}, \mathrm{kg} \cdot \mathrm{m}^{-3}\end{array}$ \\
\hline DMF1 & $1.3 \times 10^{6}$ & $1.9 \times 10^{30}$ & $8.6 \times 10^{3}$ & $7.2 \times 10^{17}$ \\
DMF2 & $9.6 \times 10^{3}$ & $1.9 \times 10^{30}$ & $8.6 \times 10^{3}$ & $7.2 \times 10^{17}$ \\
Electron-Positron & 0.51 & $6.6 \times 10^{36}$ & $2.9 \times 10^{10}$ & $6.3 \times 10^{4}$ \\
DMF3 & $3.7 \times 10^{-3}$ & $1.2 \times 10^{41}$ & $5.4 \times 10^{14}$ & $1.8 \times 10^{-4}$ \\
DMF4 & $2 \times 10^{-7}$ & $4.2 \times 10^{49}$ & $1.9 \times 10^{23}$ & $1.5 \times 10^{-21}$ \\
\hline
\end{tabular}


- DM cores of superclusters and galaxies are responsible for the gravitational lensing effect.

In WUM, the calculated maximum stellar mass is: $M_{S} \cong 174 M_{\odot}$ [40]. It is in good agreement with the mass of one of the most massive known stars R136a1: $M_{S}=215_{-31}^{+45} M_{\odot} \quad[41]$.

K. Mehrgan, et al. observed a supergiant elliptical galaxy Holmberg 15A. It has been alleged that the primary component of the galactic core is a supermassive black hole with a mass of $4 \times 10^{10} M_{\odot}$ [42]. TON 618 is a very distant and extremely luminous quasar. It possesses one of the most massive black holes ever found, with a mass of $6.6 \times 10^{10} M_{\odot}$ at the center of TON 618 [43].

How supermassive black holes initially formed is one of the biggest problems in the study of galaxy evolution today. Supermassive black holes have been observed as early as 800 million years after the Big Bang, and how they could grow so quickly remains unexplained.

C. R. Argüelles, et al. propose a novel mechanism for the creation of supermassive black holes from DM without requiring prior star formation or needing to invoke seed black holes with unrealistic accretion rates. The authors investigate the potential existence of stable galactic cores made of fermionic DM, and surrounded by a diluted dark matter halo, finding that the centers of these structures could become so concentrated that they could also collapse into supermassive black holes once a critical threshold is reached. They analyzed this mechanism with DM haloes mass up to $5.9 \times 10^{10} M_{\odot}$ [44].

According to WUM, Cores of Galaxies are DM Compact Objects made up of DMF1 and/or DMF2 with shell of DMF3 with the calculated maximum mass of $6 \times 10^{10} M_{\odot}$ (see Table 1 ). This value is in good agreement with the experimentally found values in [42] [43] and with the analyzed values in [44].

Laniakea Supercluster is a galaxy supercluster that is home to the Milky Way and approximately 100,000 other nearby galaxies. It is known as one of the largest superclusters with estimated binding mass $10^{17} M_{\odot}$ [45]. The mass-to-light ratio of the Virgo supercluster is about 300 times larger than that of the Solar ratio. Similar ratios are obtained for other superclusters [46]. In 1933, Fritz Zwicky investigated the velocity dispersion of Coma cluster and found a surprisingly high mass-to-light ratio ( 500). He concluded: "if this would be confirmed, we would get the surprising result that dark matter is present in much greater amount than luminous matter" [47]. These ratios are one of the main arguments in favor of presence of large amounts of Dark Matter in the World.

In frames of WUM, Laniakea Supercluster emerged 13.77 billion years ago due to Rotational Fission of the Supercluster Overspinning DM Core (OC) and self-annihilation of DMPs. The Core was created during Dark Epoch (spanning from the Beginning of the World for 0.45 billion years) when only Dark Matter Macroobjects existed [39].

B. Carr, F. Kühnel, and L. Visinelli "consider the observational constraints on stupendously large black holes (SLABs) in the mass range $M>10^{11} M_{\odot}$. These 
have attracted little attention hitherto, and we are aware of no published constraints on a SLAB population in the range $\left(10^{12}-10^{18}\right) M_{\odot}$. However, there is already evidence for black holes of up to nearly $10^{11} M_{\odot}$ in galactic nuclei [42], so it is conceivable that SLABs exist, and they may even have been seeded by primordial black holes" [48].

According to WUM, the calculated maximum mass of supercluster DM Core of $2.1 \times 10^{19}$ solar mass (see Table 1 ) is in good agreement with the estimated value in [45] and discussed values in [48].

In a future, these stupendously large compact objects can give rise new Luminous Superclusters as the result of their DM Cores' rotational fission. 13.77 billion years ago, the estimated number of DM Supercluster Cores in the World was around $\sim 10^{3}$ [49]. It is unlikely that all of them gave birth to Luminous Superclusters at the same cosmological time being far away from each other. In our view, there were many Beginnings for different Luminous Superclusters.

\section{Hypothesis of Hypersphere World-Universe Model}

Imagination is more important than knowledge. Knowledge is limited. Imagination encircles the world.

Albert Einstein

\subsection{Inter-Connectivity of Primary Cosmological Parameters}

The constancy of the universe fundamental constants, including Newtonian constant of gravitation, is now commonly accepted, although has never been firmly established as a fact. All conclusions on the (almost) constancy of $G$ are model-dependent. A commonly held opinion states that gravity has no established relation to other fundamental forces, so it does not appear possible to calculate it from other constants that can be measured more accurately, as is done in some other areas of physics.

WUM holds that there indeed exist relations between all Primary Cosmological Parameters (PCPs) that depend on dimensionless time-varying quantity $Q$. The Model develops a mathematical framework that allows for direct calculation of the following PCPs through $Q$ [14]:

- Newtonian parameter of gravitation $G$;

- Age of the World $A_{\tau}$;

- The Worlds' radius of curvature in the fourth spatial dimension $R$;

- Hubble's parameter $H$;

- Critical energy density $\rho_{c r}$;

- Concentration of Intergalactic Plasma $n_{I G P}$;

- Minimum Energy of Photons $E_{p h}$;

- Temperature of the Microwave Background Radiation $T_{M B R}$;

- Temperature of the Far-Infrared Background Radiation peak $T_{F I R B}$;

- Fermi coupling parameter $G_{F}$;

- Electronic neutrino rest energy $E_{v_{e}}$; 
- Muonic neutrino rest energy $E_{v_{\mu}}$;

- Tauonic neutrino rest energy $E_{v_{\tau}}$.

In frames of WUM, we calculate the values of these PCPs, which are in good agreement with the latest results of their measurements. For example:

- The predicted value of $G[50]$ :

$$
G=6.674536 \times 10^{-11} \mathrm{~m}^{3} \cdot \mathrm{kg}^{-1} \cdot \mathrm{s}^{-2}
$$

is in excellent agreement with the experimentally measured by Qing Li, et al. in 2018 values using two independent methods [51]:

$$
\begin{aligned}
& G(1)=6.674184 \times 10^{-11} \mathrm{~m}^{3} \cdot \mathrm{kg}^{-1} \cdot \mathrm{s}^{-2}(11.64 \mathrm{ppm}) \\
& G(2)=6.67484 \times 10^{-11} \mathrm{~m}^{3} \cdot \mathrm{kg}^{-1} \cdot \mathrm{s}^{-2}(11.61 \mathrm{ppm})
\end{aligned}
$$

WUM recommends the predicted value of $G$ for consideration in CODATA Recommended Values of the Fundamental Physical Constants 2022;

- The calculated value of $T_{M B R}=2.72518 \mathrm{~K}$ is in excellent agreement with experimentally measured value of $2.72548 \pm 0.00057 \mathrm{~K}$ [52];

- The calculated value of $H_{0}=68.7457 \mathrm{~km} / \mathrm{s} \cdot \mathrm{Mpc}$ is in good agreement with $H_{0}=69.32 \pm 0.8 \mathrm{~km} / \mathrm{s} \cdot \mathrm{Mpc}$ obtained using WMAP data [53] and with the newest value of

$$
H_{0}=69.6 \pm 0.8( \pm 1.1 \% \text { stat }) \pm 1.7( \pm 2.4 \% \text { sys }) \mathrm{km} / \mathrm{s} \cdot \mathrm{Mpc}
$$

found by W. L. Freedman, et al. using "the revised (and direct) measurement of the LMC (Large Magellanic Cloud) TRGB (Tip of the Red Giant Branch) extinction" [54].

The results of measurements of the Hubble's constant $H_{0}$, which characterizes the expansion rate of the universe, shows that the values of $H_{0}$ vary significantly depending on Methodology [55]. The disagreement in the values of $H_{0}$ obtained by the various teams far exceeds the standard uncertainties provided with the values. This discrepancy is called the Hubble tension.

According to WUM, the value of $H$ depends on a cosmological time only: $H=\tau^{-1}$. It means that the value of $H$ should be measured for each Galaxy separately depending on its distance to Earth and corresponding cosmological time. We must not calculate average values of $H$ depending on Methodology as it is done in experiments [55].

\subsection{Angular Momentum Problem}

Angular Momentum Problem is one of the most critical problem in Standard Cosmology (SC) that must be solved. SC does not explain how Galaxies and Extrasolar systems obtained their enormous orbital angular momenta. Any theory of evolution of the Universe that is not consistent with the Law of Conservation of Angular Momentum should be promptly ruled out.

To be consistent with this Law a Model must answer the following questions:

- How did Galaxies and Extrasolar systems obtain their substantial orbital and rotational angular momenta; 
- Why are all Macroobjects rotating;

- How did Milky Way give birth to different Extrasolar systems in different times;

- The beginning of the Milky Way (MW) galaxy was about 13.77 billion years. The age of MW is about the Age of the World. What is the origin of MW huge orbital angular momentum? We must discuss the Beginning of MW;

- The beginning of the Solar System (SS) was 4.57 billion years. What is the origin of SS orbital angular momentum? We must discuss the Beginning of SS;

- Why is the orbital momentum of Jupiter much larger than rotational momentum of the Sun. There is no possible means by which the angular momentum from the Sun could be transferred to the planets.

In our opinion, there is the only one mechanism that can provide angular momenta to Macroobjects-Rotational Fission of overspinning (surface speed at equator exceeding escape velocity) Prime objects. From the point of view of Fission model, the prime object is transferring some of its rotational angular momentum to orbital and rotational momenta of satellites. It follows that the rotational momentum of the prime object should exceed the orbital momentum of its satellite.

In frames of WUM, Prime Objects are DM Cores of Superclusters, which must accumulate tremendous angular momenta before the Birth of a Luminous World. It means that it must be some long enough time in the history of the World, which we named "Dark Epoch" [49]. To be consistent with the Law of Conservation of Angular Momentum we developed a New Cosmology of the World:

- WUM introduces Dark Epoch (spanning from the Beginning of the World for 0.45 billion years) when only DM Macroobjects (MOs) existed, and Luminous Epoch (ever since for 13.77 billion years) when Luminous MOs emerged due to the Rotational Fission of Superclusters' OCs and self-annihilation of DMPs;

- The main players of the World are Superclusters' OCs, which accumulated tremendous rotational angular momenta during Dark Epoch and transferred it to DM Cores of Galaxies during their Rotational Fission. The experimental observations of galaxies in the universe show that most of them are disk galaxies: about $60 \%$ are ellipticals and about $20 \%$ are spirals [56]. These results speak in favor of the developed Rotational Fission mechanism;

- DM Core of the Milky Way galaxy was born 13.77 billion years ago as the result of the Rotational Fission of the Virgo Supercluster DM Core;

- DM Cores of Extrasolar systems, planets and moons were born as the result of the Rotational Fissions of the Milky Way DM Core in different times (4.57 billion years ago for the Solar system);

- Macrostructures of the World form from the top (superclusters) down to galaxies, extrasolar systems, planets, and moons; 
- Gravitational waves can be a product of Rotational Fission of overspinning Macroobject Cores.

\subsection{Milky Way Centre}

The MW is a barred spiral galaxy with an estimated visible diameter of $100-200$ kly. MW is a part of the Local Group of galaxies, which form part of the Virgo Supercluster, which is itself a component of the Laniakea Supercluster. It is estimated to contain 100 - 400 billion stars. The galactic center is an intense radio source known as Sagittarius $A^{\star}\left(\operatorname{Sgr} A^{\star}\right)$, an alleged supermassive black hole of 4.268 million solar masses [57]. The oldest stars in the Milky Way are nearly as old as the universe itself and thus probably formed shortly after the Dark Ages of the Big Bang.

Several teams of researchers have attempted to image $\operatorname{Sgr} \mathrm{A}^{*}$ in the radio spectrum using very-long-baseline interferometry. The current highest-resolution ( $30 \mu \mathrm{as})$ measurement, made at a wavelength of $1.3 \mathrm{~mm}$, indicated an overall angular size for the source of $50 \mu$ as [58]. At a distance of $26.91 \mathrm{kly}=2.546 \times$ $10^{20} \mathrm{~m}$, this yields a diameter of $6 \times 10^{10} \mathrm{~m}$. Observations of a star S14 showed the mass of the object to be about 4.268 million solar masses within a volume with the radius no larger than $6.7 \times 10^{12} \mathrm{~m}$ [57].

In 2010, two gigantic spherical bubbles of high energy emission were detected to the north and the south of the MW core, using data from the Fermi Gamma-ray Space Telescope. The diameter of each of the bubbles is about $25 \mathrm{kly}$ (see Section 4.4).

In 2015, NASA reported observing an X-ray flare 400 times brighter than usual, a record-breaker, from Sgr $A^{\star}$. The unusual event may have been caused by the entanglement of magnetic field lines within gas flowing into Sgr $A^{\star}$ [59].

E. A. C. Mills in her "Journey to the Center of the Galaxy: Following the gas to understand past and future activity in galaxy nuclei" wrote [60]:

"The young stars in the central lightyear, the innermost of whose orbits are famously used to determine parameters of central supermassive black hole, are suggested to have formed in-situ in one of the most extreme environments imaginable: in an incredibly dense gas disk a fraction of a light year from the black hole. Even allowing for recent activity in the past few hundred years which we can detect from the $X$-ray light of these outbursts reflecting off of clouds a few hundred light years from the black hole... our black hole is no AGN' (Active Galactic Nucleus).

Prof. R. Genzel and A. Ghez were awarded the 2020 Nobel Prize in Physics for their discovery that $\mathrm{Sgr}^{*}$ is a supermassive compact object, for which a black hole is the only currently accepted explanation.

In 2013, we proposed a principally different explanation of supermassive compact objects: "Macroobjects of the World have cores made up of the discussed DM particles. Other particles, including DM and baryonic matter, form shells surrounding the cores" [40]. 
In frames of WUM (see Table 1):

- The calculated value of the radius of the Electron-Positron shell $2.9 \times 10^{10} \mathrm{~m}$ is in excellent agreement with the experimentally measured value of the radio source $3 \times 10^{10} \mathrm{~m}[58]$;

- The calculated value of the mass of the Electron-Positron shell $6.6 \times 10^{36} \mathrm{~kg}$ is in good agreement with the experimentally measured value of the supermassive compact object $8.5 \times 10^{36} \mathrm{~kg}$ [57];

- The additional mass of the DMF3 shell of $1.9 \times 10^{36} \mathrm{~kg}$ is much smaller than the maximum mass of $1.2 \times 10^{41} \mathrm{~kg}$;

- X-ray flare 400 times brighter than usual can be explained by DMF3 particles (3.7 keV) self-annihilation [59];

- The excess of gamma-ray emission with energy about $10 \mathrm{GeV}$ reported by D. Hooper from the Galactic Center [61] can be explained by DMF2 particles $(9.6 \mathrm{GeV})$ self-annihilation;

- Bipolar astrophysical gamma-ray jets [62] can be explained by DMF1 (1.3 $\mathrm{TeV})$ and DMF2 particles $(9.6 \mathrm{GeV})$ self-annihilation;

- The Galactic Centre "chimneys" [63] are the result of the self-annihilation of DMF3 (3.7 keV);

- DM Fermi Bubbles can be explained based on DMF1, DMF2, and DMF3 particles (see Section 4.4).

The oldest known star HD 140283 (Methuselah star) is a subgiant star about 190 light years away from Earth for which a reliable age has been determined [64]. H. E. Bond, et al. found its age to be $14.46 \pm 0.8 \mathrm{Byr}$ that does not conflict with the Age of the Universe, $13.77 \pm 0.06 \mathrm{Byr}$, based on the microwave background radiation and Hubble constant [53]. It means that this star must have formed between 13.66 and 13.83 Byr, an amount of time that is too short for formation of the second generation of stars according to prevailing theories. In WUM, this discovery can be explained by generation of HD 140283 by overspinning Core of MW 13.77 billion years ago.

In frames of the developed Rotational Fission model, it is easy to explain hyper-runaway stars unbound from MW with speeds of up to $~ 700 \mathrm{~km} / \mathrm{s}$ [65]: they were launched by overspinning Core of the Large Magellanic Cloud with the speed higher than the escape velocity.

S. E. Koposov, et al. present the discovery of the fastest Main Sequence hyper-velocity star S5-HVS1 with mass of about 2.3 solar mass that is located at a distance of $\sim 9 \mathrm{kpc}$ from the Sun. When integrated backwards in time, the orbit of the star points unambiguously to the Galactic Centre, implying that S5-HVS1 was kicked away from Sgr $A^{\star}$ with a velocity of $\sim 1800 \mathrm{~km} / \mathrm{s}$, and travelled for 4.8 Myr to its current location. So far, this is the only hyper-velocity star confidently associated with the Galactic Centre [66]. In frames of the developed Model, this discovery can be explained by Gravitational Burst (GB) of the overspinning Core of the Milky Way 4.8 million years ago, which gave birth to S5-HVS1 with the speed higher than the escape velocity of the Core. 
C. J. Clarke, et al. observed CI Tau, a young 2-million-year-old star. CI Tau is located about 500 light years away in a highly-productive stellar "nursery" region of the galaxy. They discovered that the Extrasolar system contains four gas giant planets that are only 2 million years old [67], an amount of time that is too short for formation of gas giants according to the prevailing theories. In frames of the developed Rotational Fission model, this discovery can be explained by GB of the MW Core 2 million years ago, which gave birth to the CI Tau system with all the planets generated at the same time.

The totality of the obtained experimental results testify in favor of the existence of the supermassive compact object made of DMPs at the Milky Way Centre.

\subsection{Dark Matter Fermi Bubbles}

In 2010, the discovery of two Fermi Bubbles (FBs) emitting gamma- and X-rays was announced. FBs extend for about $25 \mathrm{kly}$ above and below the center of the galaxy [68]. The outlines of the bubbles are quite sharp, and the bubbles themselves glow in nearly uniform gamma rays over their colossal surfaces. Gamma-ray spectrum at Galactic latitude $\leq 10^{\circ}$, without showing any sign of cutoff up to around $1 \mathrm{TeV}$, remains unconstrained [69]. Years after the discovery of FBs, their origin and the nature of the gamma-ray emission remain unresolved.

WUM explains FBs the following way [39]:

- Core of the Milky Way is made up of DMPs: DMF1 (1.3 TeV), DMF2 (9.6 $\mathrm{GeV}$ ), and DMF3 (3.7 keV). The second component (DMF2) explains the excess $\mathrm{GeV}$ emission reported by Dan Hooper from the Galactic Center [61]. Core rotates with surface speed at equator close to the escape velocity between Gravitational Bursts (GBs), and over the escape velocity at the moments of GBs;

- Bipolar astrophysical jets (which are astronomical phenomena where outflows of matter are emitted as the extended beams along the axis of rotation [70]) of DMPs are ejected from the rotating Core into the Galactic halo along the rotation axis of the DM Core;

- Due to self-annihilation of DMF1 and DMF2, these beams are gamma-ray jets [62]. The prominent X-ray structures on intermediate scales (hundreds of parsecs) above and below the plane (named the Galactic Centre "chimneys" [63]) are the result of the self-annihilation of DMF3 particles;

- FBs are bubbles whose boundary with the Intergalactic Medium has a basic surface energy density $\sigma_{0}$ equals to: $\sigma_{0}=h c / a^{3}$. These bubbles are filled with DMPs: DMF1, DMF2, and DMF3. The calculated diameter $D_{F B}$ of FBs: $D_{F B}=28.6 \mathrm{kly}$ is in good agreement with the measured size of the FBs $25 \mathrm{kly}$ [68] and $32.6 \mathrm{kly}$ [63]. FBs made up of DMF3 particles resemble a honeycomb filled with DMF1 and DMF2;

- With Nikola Tesla's principle at heart- “There is no energy in matter other than that received from the environment"-we calculate mass $M_{F B}$ of FBs: 
$M_{F B}=3.6 \times 10^{41} \mathrm{~kg}$. Recall that the mass of MW $M_{M W}$ is about:

$M_{M W}=(1.6-3.2) \times 10^{42} \mathrm{~kg}$;

- FBs radiate X-rays due to the self-annihilation of DMF3 (3.7 keV). Gamma rays up to $1 \mathrm{TeV}$ [71] are the result of self-annihilation of DMF1 $(1.3 \mathrm{TeV})$ and DMF2 $(9.6 \mathrm{GeV})$ particles in Dark Matter Objects (DMOs) whose density is sufficient for the self-annihilation of DMPs to occur. On the other hand, DMOs are much smaller than stars in the World, and have a high concentration in FBs to provide nearly uniform gamma ray glow over their colossal surfaces [39];

- The total flux of the gamma radiation from FBs is the sum of the contributions of all individual DMOs, which irradiate gamma quants with different energies and attract new DMF1 and DMF2 particles from FBs. The Core of MW supplies FBs with new DMPs through the galactic wind, explaining the brightness of FBs remaining fairly constant during the time of observations. In our opinion, FBs are built continuously throughout the lifetime of the Milky Way galaxy.

In our view, Fermi Bubbles are DMPs' clouds containing uniformly distributed Dark Matter Objects, in which DMPs self-annihilate and radiate Xrays and gamma rays. DM Fermi Bubbles constitute a principal proof of WUM.

\subsection{Dark Matter Reactors}

The following facts support the existence of Dark Matter Cores in Macroobjects:

- E. Fossat, et al. found that Solar Core rotates $3.8 \pm 0.1$ faster than the surrounding envelope [72];

- By analyzing the earthquake doublets, J. Zhang, et al. concluded that the Earth's inner core is rotating faster than its surface by about $0.3-0.5$ degrees per year [73];

- T. Guillot, et al. found that a deep interior of Jupiter rotates nearly as a rigid body, with differential rotation decreasing by at least an order of magnitude compared to the atmosphere [74].

The fact that Macroobject Cores rotate faster than surrounding envelopes, despite high viscosity of an internal medium, is intriguing. WUM explains this phenomenon through absorption of DMPs by Cores. DMPs supply not only additional mass $\left(\propto \tau^{3 / 2}\right)$, but also additional angular momentum $\left(\propto \tau^{2}\right)$. Cores irradiate products of self-annihilation, which carry away excessive angular momentum. The Solar wind is the result of this mechanism [39].

$\mathrm{W}$. $\mathrm{Wu}, \mathrm{S} . \mathrm{Ni}$, and J. Irving investigated scattered seismic waves traveling inside the Earth to constrain the roughness of the Earth's $660-\mathrm{km}$ boundary [75]. The researchers were surprised by just how rough that boundary is - rougher than the surface layer that we all live on. The roughness was not equally distributed, either; just as the crust's surface has smooth ocean floors and massive mountains, the 660-km boundary has rough areas and smooth patches [76].

$\mathrm{X}$. Markenscoff in the paper "Volume collapse' instabilities in deep-focus 
earthquakes: A shear source nucleated and driven by pressure" explains "the mystery of the long-standing observations in deep-focus earthquakes (400 - 700 $\mathrm{km}$ ) by symmetry-breaking instabilities in high-pressure phase transformation, which produce the counterintuitive phenomenon of "volume collapse" producing only shear radiation, with little, or no, volumetric component, even under conditions of full isotropy" [77].

According to WUM, the $660-\mathrm{km}$ boundary is a boundary between Earth's DM core and Upper mantle with Crust, which were produced by DM core during 4.57 billion years [49]. The deep-focus earthquakes are connected with random mass ejections happening at the $660-\mathrm{km}$ boundary as the result of DMPs selfannihilation in the DM core.

Random Variations of Earth's Rotational Speed. G. Jones and K. Bikos in the paper "Earth Is in a Hurry in 2020" wrote [78]: "When highly accurate atomic clocks were developed, they showed that the length of a mean solar day can vary by milliseconds. These differences are obtained by measuring the Earth's rotation with respect to distant astronomical objects'. It turned out that the variations of the daylength throughout 2020 were in the range $86400_{-1.46 \mathrm{~ms}}^{+1.62 \mathrm{ss}} \mathrm{s}$.

Jean-Luc Margot, et al. observed the analogous effect on Venus. The average sidereal day on Venus in the 2006-2020 interval is $243.0226 \pm 0.0013$ Earth days [79].

In frames of WUM, Random variations of the Earth's and Venus's rotational speed on a daily basis can be explained by variations in an activity of the Earth's and Venus's Dark Matter Reactors (DMRs). As the result of DMPs self-annihilation, random mass ejections are happening. During a time of high DMR activity, the Earth's and Venus's rotational speed is lower (long days) due to increase of their moment of inertia. When random mass ejections are less frequent, the Earth's and Venus's moment of inertia is decreasing, we observe short days.

Gravitationally-Rounded Objects Internal Heat. The analysis of Sun's heat for planets in Solar system yields the effective temperature of Earth of $255 \mathrm{~K}$ [80]. The actual mean surface temperature of Earth is $288 \mathrm{~K}$ [81]. The higher actual temperature of Earth is due to energy generated internally by the planet itself. According to the standard model, the Earth's internal heat is produced mostly through radioactive decay. The major heat-producing isotopes within Earth are K-40, U-238, and Th-232. Radiogenic decay can be estimated from the flux of geoneutrinos that are emitted during radioactive decay. Based on the observations the KamLAND Collaboration made a conclusion that "heat from radioactive decay contributes about half of Earth's total heat flux" [82];

Jupiter radiates more heat than it receives from the Sun [83]. Giant planets like Jupiter are hundreds of degrees warmer than current temperature models predict. Until now, the extremely warm temperatures observed in Jupiter's atmosphere (about 970 degrees C [84]) have been difficult to explain, due to lack of a known heat source. Saturn radiates 2.5 times more energy than it receives from the Sun [85]; Uranus-1.1 times [86]; Neptune-2.6 times [87]. Many Icy Solar system bodies including Pluto possess subsurface oceans [88]. 
According to WUM, the internal heating of all gravitationally-rounded objects of the Solar system is due to DMPs self-annihilation in their cores made up of DMF1 (1.3 TeV). The amount of energy produced due to this process is sufficiently high to heat up the objects. New DMF1 freely penetrate through the entire objects' envelope, get absorbed into the cores, and continuously support DMF1 self-annihilation.

Half of the chemical elements heavier than iron are produced by the rapid neutron capture process (r-process), which produces many of the heavy chemical elements, but the astrophysical settings where it occurs remain unclear. Plutonium-244 with half-life of 80.6 million years and Iron-60 with half-life of 2.6 million years are not produced in significant quantities by the nuclear fuel cycle, because it needs very high neutron flux environments. Any Pu-244 and Iron-60 present in the Earth's crust should have decayed by now. Nevertheless, D. C. Hoffman, et al. in 1971 obtained the first indication of $\mathrm{Pu}-244$ present existence in Nature [89].

In 2021, A. Wallner, et al. analyzed the plutonium content of a deep-sea crust sample, "identifying a few dozen atoms of the r-process isotope Pu-244 that were delivered to Earth within the past few million years. There was a simultaneous signal of iron-60, which is known to be produced in supernovae. Comparing the ratios of these isotopes constrains the relative contributions of supernovae and neutron star mergers to r-process nucleosynthesis" [90].

In frames of WUM, all chemical products of the Earth including isotopes $\mathrm{K}-40, \mathrm{U}-238$, Th-232, $\mathrm{Pu}-244$, and Iron-60, are produced within the Earth as the result of DMF1 particles self-annihilation. They arrive to the Crust of the Earth due to convection currents in the mantle carrying heat and isotopes from the interior to the planet's surface [91].

Dark Matter Reactors. Macroobjects' cores are essentially Dark Matter Reactors fueled by DMPs. All chemical elements, compositions, radiations are produced by Macroobjects themselves as the result of DMPs self-annihilation and an uncontrolled thermonuclear fusion of them into heavier Dark Matter Superparticles (DMSPc) within their cores. The diversity of all gravitationally-rounded objects in the Solar system is explained by the differences in their DM cores (mass, size, density, composition). The DM Reactors at their cores (including Earth) are very efficient and provide enough energy for the internal heating and all their geological processes like volcanos, quakes, mountains' formation through tectonic forces or volcanism, tectonic plates' movements, etc.

Scientists from the Tibet AS $\gamma$ experiment observed gamma rays with energies between 0.1 and $1 \mathrm{PeV}$, coming from the galactic disk regions. Specifically, they found 23 ultra-high-energy cosmic gamma rays with energies above $398 \mathrm{TeV}$ along MW [92].

In frames of WUM, the gamma rays with energies between $1 \mathrm{TeV}$ and $1 \mathrm{PeV}$ can be explained by nuclear fission of DMSPs, consisting of many fused DMF1 $(1.3 \mathrm{TeV})$, produced in the cores of MW and stars. 


\subsection{Solar Corona, Geocorona, Planetary Coronas}

Structure of Solar Atmosphere. According to the standard model, the visible surface of the Sun, the photosphere, is the layer below which the Sun becomes opaque to visible light [93]. Above the photosphere visible sunlight is free to propagate into space, and almost all of its energy escapes the Sun entirely. The sunlight has the spectrum of a black-body radiating at about $5800 \mathrm{~K}$.

Above the photosphere lies the chromosphere that is about $2500 \mathrm{~km}$ thick, dominated by a spectrum of emission and absorption lines. The temperature of the chromosphere increases gradually with altitude, ranging up to $\sim 2 \times 10^{4} \mathrm{~K}$ near the top. The particle density decreases rapidly from $10^{22}$ to $10^{17} \mathrm{~m}^{-3}$ [94] [95].

Above the chromosphere, in a thin (about $200 \mathrm{~km}$ ) transition region, the temperature rises rapidly to coronal temperatures closer to $10^{6} \mathrm{~K}$. The particle density decreases from $10^{17}$ up to $10^{16}-10^{15} \mathrm{~m}^{-3}$ in the low corona [94].

Solar Corona is an aura of plasma that surrounds the Sun and extends at least $8 \times 10^{6} \mathrm{~km}$ into outer space [96] (compare with the Sun's radius $7 \times 10^{5} \mathrm{~km}$ ). Spectroscopy measurements indicate strong ionization and plasma temperature in excess of $10^{6} \mathrm{~K}$ [97]. The corona emits radiation mainly in the X-rays, observable only from space. The plasma is transparent to its own radiation and to solar radiation passing through it, therefore we say that it is optically-thin. The gas, in fact, is very rarefied, and the photon mean free-path by far overcomes all other length-scales, including the typical sizes of the coronal features.

J. Schmelz made the following comment on the composition of Solar corona: "Along with temperature and density, the elemental abundance is a basic parameter required by astronomers to understand and model any physical system. The abundances of the solar corona are known to differ from those of the solar photosphere" [98].

Coronal Heating Problem in solar physics relates to the question of why the temperature of the Solar corona is millions of degrees higher than that of the photosphere. The high temperatures require energy to be carried from the solar interior to the corona by non-thermal processes.

According to WUM, the origin of the Solar corona plasma is not the coronal heating. Plasma particles (electrons, protons, multicharged ions) are so far apart that plasma temperature in the usual sense is not very meaningful. The plasma is the result of a self-annihilation of DMF1 (1.3 TeV), DMF2 (9.6 GeV), and DMF3 $(3.7 \mathrm{keV})$ particles. The Solar corona made up of DMPs resembles a honeycomb filled with plasma.

The following experimental results speak in favor of this model [39]:

- The corona emits radiation mainly in X-rays due to the self-annihilation of DMF3 particles;

- The plasma is transparent to its own radiation and to the radiation coming from below;

- The elemental composition of the Solar corona and the Solar photosphere are 
known to differ;

- During the impulsive stage of Solar flares, radio waves, hard x-rays, and gamma rays with energy above $100 \mathrm{GeV}$ are emitted [99] (one photon had an energy as high as $467.7 \mathrm{GeV}$ [49]). In our view, it is the result of enormous density fluctuations of DMPs in the Solar corona and their self-annihilation;

- Assuming the particle density in the low corona $10^{15} \mathrm{~m}^{-3}$ and mass of DMF1 particles: $m_{D M F 1}=2.3 \times 10^{-24} \mathrm{~kg}$ we can find mass density $\rho_{D M F 1}^{\text {in }}=2.3 \times 10^{-9} \mathrm{~kg} / \mathrm{m}^{3}$ that is equal to the density of the fractal structure [49];

- A distance between DMF1 particles is about $10^{-5} \mathrm{~m}$ that is much smaller than the range of the introduced in WUM weak interaction of DMPs:

$R_{W}=1.65314 \times 10^{-4} \mathrm{~m}$ [39]. Weak Interaction between DMPs provides integrity of a Solar corona;

- At the same density of the fractal structure, a distance between DMF3 particles with mass $m_{D M F 3}=6.7 \times 10^{-33} \mathrm{~kg}$ is about $10^{-8} \mathrm{~m}$. The smallest distance between DMF3 explains the fact that corona emits radiation mainly in the $\mathrm{X}$-rays;

- The Solar corona is a stable Shell around the Sun with the inner radius $R_{\text {in }} \cong 7 \times 10^{8} \mathrm{~m}$ and an outer radius $R_{\text {out }} \cong 3 \times 10^{12} \mathrm{~m}$. The total mass of the Corona is: $M_{S C} \cong 9 \times 10^{25} \mathrm{~kg}$ [49];

- Observable outer radius of the Solar corona $8 \times 10^{9} \mathrm{~m}$ [96] depends on the concentration of DMPs, the strength of their self-annihilation interaction, and a sensitivity of the measuring instrument.

Geocorona is a luminous part of an outermost region of the Earth's atmosphere that extends to at least $640,000 \mathrm{~km}$ from the Earth [100]. It is seen primarily via Far-Ultra-Violet (FUV) light from the Sun that is scattered by neutral hydrogen [101]. The first high-quality and wide-field-of-view image of Earth's corona of $243,000 \mathrm{~km}$ was obtained by the first interplanetary microspacecraft. It acquires spectral images (52-148 nm) of the atmospheres of planets from Earth orbit and has provided quasi-continuous remote sensing observations of the geocorona since 2013 [102]. The most popular explanation of this geocoronal emission is a scattering of Solar FUV photons by exospheric hydrogen [103].

$\mathrm{X}$-rays from Earth's Geocorona were first detected by Chandra X-ray Observatory in 1999 [104]. X-rays were observed in the range of energies 0.08 - 10 $\mathrm{keV}$. The main mechanism explaining the geocoronal X-rays is that they are caused by collisions between neutral atoms in the geocorona with carbon, oxygen and nitrogen ions that are streaming away from the Sun in the solar wind [104] [105] [106]. This process is called "charge exchange" since an electron is exchanged between neutral atoms in geocorona and ions in the solar wind.

X-rays from Planets were also observed by Chandra [104]. According to NASA:

- The X-rays from Venus and, to some extent, the Earth, are due to the fluorescence of solar X-rays striking the atmosphere; 
- Fluorescent X-rays from oxygen atoms in the Martian upper atmosphere are similar to those on Venus. A huge Martian dust storm was in progress when the Chandra observations were made. The intensity of the X-rays did not change during the dust storm;

- Jupiter has an environment capable of producing X-rays in a different manner because of its substantial magnetic field. X-rays are produced when highenergy particles from the Sun get trapped in its magnetic field and accelerated toward the polar regions where they collide with atoms in Jupiter's atmosphere;

- Like Jupiter, Saturn has a strong magnetic field, so it was expected that Saturn would also show a concentration of X-rays toward the poles. However, Chandra's observation revealed instead an increased X-ray brightness in an equatorial region. Furthermore, Saturn's X-ray spectrum was found to be similar to that of X-rays from Sun.

In our opinion, the Planetary Coronas are similar to the Solar Corona [39]:

- At the distance of $640,000 \mathrm{~km}$ from the Earth [87], atoms and molecules are so far apart that they can travel hundreds of kilometers without colliding with one another. Thus, the exosphere no longer behaves like a gas, and the particles constantly escape into space. In our view, FUV radiation and X-rays are the consequence of DMF3 particles self-annihilation;

- All planets and some observed moons (Europa, Io, Io Plasma Torus, Titan) have X-rays in upper atmosphere of the planets, similar to the Solar Corona;

- The Geocorona is a stable Shell around the Earth with inner radius $R_{\text {in }} \cong 6.5 \times 10^{3} \mathrm{~km}$ and observed outer radius $R_{\text {out }} \cong 6.4 \times 10^{5} \mathrm{~km}$. The total mass of this Shell is: $M_{G C} \cong 4.1 \times 10^{18} \mathrm{~kg}$.

\section{Hypersphere World-Universe Model}

\subsection{Assumptions}

WUM is based on three primary assumptions:

- The World is a finite 3D Hypersphere of a $4 \mathrm{D}$ Nucleus of the World that is expanding along the fourth spatial dimension of the Nucleus with speed equals to the gravitodynamic constant $c$. The Universe serves as an unlimited source of DM, which continuously created in the Nucleus of the World. Ordinary Matter is a by-product of DMPs self-annihilation;

- Medium of the World, consisting of protons, electrons, photons, neutrinos, and DMPs, is an active agent in all physical phenomena in the World;

- Two fundamental parameters in various rational exponents define all macro and micro features of the World: dimensionless Rydberg constant $\alpha$ and dimensionless quantity $Q$ that is a measure of the Size $R$ and Age $A_{\tau}$ of the World and is, in fact, the Dirac Large Number.

\subsection{Evidence of Hypersphere World}

The physical laws we observe appear to be independent of the Worlds' curvature 
in the fourth spatial dimension due to the very small value of the dimensiontransposing gravitomagnetic parameter of the Medium [107]. Consequently, direct observation of the Worlds' curvature would appear to be a hopeless goal.

One way to prove the existence of the Worlds' curvature is direct measurement of truly large-scale parameters of the World: Gravitational, Hubble's, Temperature of the Microwave Background Radiation. Conducted at various points of time, these measurements would give us varying results, providing insight into the curved nature of the World. Unfortunately, the accuracy of the measurements is quite poor. Measurement errors far outweigh any possible "curvature effects", rendering this technique useless in practice. To be conclusive, the measurements would have to be conducted billions of years apart [108].

Let's consider the so-called Faint Young Sun problem, an effect that has indeed been observed for billions of years, albeit indirectly [15]. 4.57 billion years ago the Sun's output has been only $70 \%$ as intense as it is today [93]. One of the consequences of WUM holds that all stars were fainter in the past. As their cores absorb new DM, size of macroobjects cores $R_{M O}$ and their luminosity $L_{M O}$ are increasing in time $R_{M O} \propto Q^{1 / 2} \propto \tau^{1 / 2}$ and $L_{M O} \propto Q \propto \tau$, respectively. Taking the Age of the World $\cong 14.22 \mathrm{Byr}$ and the age of the Solar system $\cong 4.57 \mathrm{Byr}$, it is easy to find that the young Suns' output was $67 \%$ of what it is in the present epoch.

In WUM, Local Physics is linked with the large-scale structure of the Hypersphere World through the dimensionless quantity $Q$. The proposed approach to the fourth spatial dimension agrees with Mach's principle: "Local physical laws are determined by the large-scale structure of the universe". Applied to WUM, it follows that all parameters of the World depending on $Q$ are a manifestation of the Worlds' curvature in the fourth spatial dimension of the Nucleus of the World [15].

\subsection{Principal Points}

The Beginning. The World was started by a fluctuation in the Eternal Universe, and the Nucleus of the World, which is a $4 \mathrm{D}$ ball, was born. An extrapolated Nucleus radius at the Beginning was equal to the basic unit of size $a$. The World is the 3D Finite Boundless Hypersphere that is the surface of the 4D Nucleus. All points of the Hypersphere are equivalent; there are no preferred centers or boundaries of the World. The extrapolated energy density of the World at the Beginning was four orders of magnitude smaller than the nuclear energy density.

Expansion. The 4D Nucleus is expanding along Its fourth spatial dimension and Its surface, the 3D Hypersphere, is likewise expanding so that the radius of the Nucleus is increasing with speed $c$ that is the gravitodynamic constant.

Creation of Matter. The surface of the Nucleus is created in a process analogous to sublimation. DM is created by the Universe in the 4D Nucleus of the World. DMPs carry new DM into the 3D Hypersphere World. Ordinary Matter is a byproduct of DMPs self-annihilation. Consequently, the matter-antimatter 
asymmetry problem discussed in literature does not arise. Creation of Matter is a direct consequence of expansion.

Content of the World. The World consists of the Medium and Macroobjects (MOs). Total energy density of the World equals to the critical energy density throughout the World's evolution. The energy density of the Medium is $2 / 3$ of the total energy density and MOs (Galaxy clusters, Galaxies, Extrasolar systems, Planets, Moons, etc.) $-1 / 3$ in all cosmological times. The relative energy density of DMF4 particles is about 68.8\%, self-annihilating DMPs (DMF1, DMF2, DMF3, DIRACs, and ELOPs) - about 24\%, and Ordinary Particles (protons, electrons, photons, and neutrinos)-about 7.2\%.

Two Fundamental Parameters in various rational exponents define all $\mathrm{mi}$ cro- and macro-features of the World: dimensionless Rydberg constant $\alpha$ and Quantity $Q$. The World's energy density is proportional to $Q^{-1}$ in all cosmological times. Particles relative energy densities are proportional to $\alpha$. $Q$ in present epoch equals to: $Q=0.759972 \times 10^{40}$.

Supremacy of Matter. Time, Space and Gravitation have no separate existence from Matter. They are closely connected with the Impedance, Gravitomagnetic parameter, and Energy density of the Medium, respectively.

Inter-Connectivity of Primary Cosmological Parameters. WUM reveals the Inter-Connectivity of PCPs and calculates their values, which are in good agreement with the latest results of their measurements.

WUM introduces Dark Epoch (spanning from the Beginning of the World for 0.45 billion years) and Luminous Epoch (ever since, 13.77 billion years). Transition from Dark Epoch to Luminous Epoch is due to Rotational Fission of Overspinning DM Supercluster's Cores and self-annihilation of DMPs.

Macroobjects Shell Model. Macroobjects of the World possess the following properties: their Cores are made up of DMPs; they contain other particles, including DMPs and Ordinary Particles, in shells surrounding the Cores. Introduced Weak Interaction between DMPs provides integrity of all shells. Self-annihilation of DMPs can give rise to any combination of gamma- and X-ray lines.

Macroobjects Formation and Evolution. Macroobjects form from superclusters down to galaxies and extrasolar systems in parallel around different Cores made up of different DMPs. Formation of galaxies and stars is not a process that concluded ages ago; instead, it is ongoing. Assuming an Eternal Universe, the numbers of cosmological structures on all levels will increase; new galaxy clusters will form; existing clusters will obtain new galaxies; new stars will be born inside existing galaxies; sizes of individual stars will increase, etc. The temperature of the Medium will asymptotically approach absolute zero.

Nucleosynthesis of all elements occurs inside of Macroobjects during their evolution. Stellar nucleosynthesis theory should be enhanced to account for selfannihilation of DMPs inside of Stars.

Black-body spectrum of the Cosmic Microwave Background Radiation is due to thermodynamic equilibrium of photons with Intergalactic Plasma. 
Milky Way Galaxy is a Disk Bubble whose boundary with Intergalactic Medium has a surface energy density $\sigma_{0}$. The Disk Bubble contains Intragalactic Medium and (100 - 400) billion Stars.

DM Fermi Bubbles are stable clouds of DMPs containing uniformly distributed Dark Matter Objects, in which DMPs self-annihilate and radiate X-rays and gamma rays. Proposed Weak interaction between particles DMF3 (3.7 keV) provides integrity of Fermi Bubbles.

Extrasolar Systems. The boundary between Extrasolar systems and Intragalactic Medium has a surface energy density $\sigma_{0}$. This bubble-like region of space, which surrounds the Sun, is named Heliosphere that is continuously inflated by Solar jets, known as the Solar wind.

Solar System. A detailed analysis of the Solar system shows that the overspinning DM Core of the Sun can give birth to DM planetary cores, and they can generate DM cores of moons through the Rotational Fission mechanism.

Solar Corona, Geocorona and Planetary Coronas made up of DMPs resemble honeycombs filled with plasma particles (electrons, protons, and multicharged ions), which are the result of DMPs self-annihilation.

Lightning Initiation problem and Terrestrial Gamma-Ray Flashes are explained by self-annihilation of DMPs in Geocorona.

Dark Matter Reactors. Macroobjects' cores are essentially Dark Matter Reactors fueled by DMPs. All chemical elements, compositions, radiations are "Homemade" and produced by Macroobjects themselves as the result of DMPs selfannihilation in their DM cores.

\subsection{Predictions}

It does not make any difference how beautiful your guess is, it doesn't make any difference how smart you are, who made the guess, or what his name is.

If it disagrees with experiment, it's wrong. That's all there is to it.

Richard Feynman

In 2013, WUM revealed a self-consistent set of time-varying values of Primary Cosmological Parameters of the World: Gravitation parameter, Hubble's parameter, Age of the World, Temperature of Microwave Background Radiation, and concentration of Intergalactic plasma. Based on the inter-connectivity of these parameters, WUM solved the Missing Baryon problem and predicted the values of the following Cosmological parameters: gravitation, concentration of Intergalactic plasma, and the minimum energy of photons [40], which were experimentally confirmed in 2015-2018.

The results obtained by K. Mehrgan, et al. [42] and O. Shemmer, et al. [43]; discussed by C. R. Argüelles, et al. [44] and B. Carr, et al. [46]; and "The Discovery of a Supermassive Compact Object at the Centre of Our Galaxy" (Nobel Prize in Physics 2020) made by R. Genzel and A. Ghez confirm one of the most important predictions of WUM in 2013: "Macroobjects of the World have cores made up of the discussed DM particles. Other particles, including DM and baryonic matter, form shells surrounding the cores" [40]. 


\section{Conclusion}

The Hypersphere World-Universe Model successfully describes primary cosmological parameters and their relationships, ranging in scale from cosmological structures to elementary particles. WUM allows for precise calculation of values that were only measured experimentally earlier and makes verifiable predictions. WUM does not attempt to explain all available cosmological data, as that is an impossible feat for any one manuscript. Nor does WUM pretend to have built all-encompassing theory that can be accepted as is. The Model needs significant further elaboration, but in its present shape, it can already serve as a basis for a New Cosmology proposed by Paul Dirac in 1937. The Model should be developed into a well-elaborated theory by entire physical community.

\section{Acknowledgements}

I am eternally grateful to my Scientific Father Paul Dirac who was a genius and foresaw the Future of Physics in a New Cosmology. I am much obliged to Prof. C. Corda for publishing my manuscripts in JHEPGC. Special thanks to my son I. Netchitailo who helped me refine the Model and improve its understanding.

\section{Conflicts of Interest}

The author declares no conflicts of interest regarding the publication of this paper.

\section{References}

[1] Netchitailo, V. (2018) Hypersphere World-Universe Model. Tribute to Classical Physics. Journal of High Energy Physics, Gravitation and Cosmology, 4, 441-470. https://doi.org/10.4236/jhepgc.2018.43024

[2] Maxwell, J.C. (1861) On Physical Lines of Force. Philosophical Magazine, 90, 11-23. https://doi.org/10.1080/14786431003659180

[3] Kohlrausch, R. and Weber, W. (1857) Elektrodynamische Maaßbestimmungen: Insbesondere Zurückfuhrung der Stromintensitäts-Messungen auf mechanisches Maass. On the Amount of Electricity which Flows through the Cross-Section of the Circuit in Galvanic Currents (Translated by Susan P. Johnson and Edited by Laurence Hecht). http://ppp.unipv.it/Collana/Pages/Libri/Saggi/Volta\%20and\%20the $\% 20$ History\%20 of\%20Electricity/V\%26H\%20Sect3/V\%26H\%20287-297.pdf

[4] Fizeau, H. (1849) Comptes Rendus: Hebdomadaires de scéances de l'Academie de Sciences. Paris, 29, 90-92.

[5] Maxwell, J.C. (1865) A Dynamical Theory of the Electromagnetic Field. Philosophical Transactions of the Royal Society of London, 155, 459-512. https://doi.org/10.1098/rstl.1865.0008

[6] Heüman, G.D. (1888) The Rydberg Formula as Presented to Matematiskt-Fysiska Förening. https://www.cosmos-indirekt.de/Physik-Schule/Rydberg-Formel

[7] Thomson, J.J. (1897) Cathode Rays. Philosophical Magazine, 44, 293-316. http://web.lemoyne.edu/ giunta/thomson1897.html

[8] Plank, M. (1901) On the Law of Distribution of Energy in the Normal Spectrum. Annalen der Physik, 4, 553-563. https://doi.org/10.1002/andp.19013090310 
https://web.archive.org/web/20081217042934/http://dbhs.wvusd.k12.ca.us/webdocs /Chem-History/Planck-1901/Planck-1901.html

[9] McCullagh, J. (1846) An Essay towards a Dynamical Theory of Crystalline Reflexion and Refraction. Transactions of the Royal Irish Academy, 21, 17-50.

[10] Tesla, N. (1937) Prepared Statement on the 81st Birthday Observance. http://www.institutotesla.org/tech/TeslaGravity.html

[11] Dirac, P.M. (1951) Is There an Aether? Nature, 168, 906-907.

[12] Netchitailo, V.S. (2016) 5D World-Universe Model. Gravitation. Journal of High Energy Physics, Gravitation and Cosmology, 2, 328-343.

https://doi.org/10.4236/jhepgc.2016.23031

[13] Riemann, B. (1854) On the Hypotheses Which Lie at the Bases of Geometry (Translated by William Kingdon Clifford). Nature, 8, 36-37.

[14] Netchitailo, V.S. (2017) Mathematical Overview of Hypersphere World-Universe Model. Journal of High Energy Physics, Gravitation and Cosmology, 3, 415-437. https://doi.org/10.4236/jhepgc.2017.33033

[15] Netchitailo, V.S. (2016) Overview of Hypersphere World-Universe Model. Journal of High Energy Physics, Gravitation and Cosmology, 2, 593-632. https://doi.org/10.4236/jhepgc.2016.24052

[16] Thirring, H. (1918) On the Formal Analogy between the Basic Electromagnetic Equations and Einstein's Gravity Equations in First Approximation. Physikalische Zeitschrift, 19, 204-205.

[17] Heaviside, O. (1893) A Gravitational and Electromagnetic Analogy. The Electrician, 31, 281-282.

[18] Xu, G.C. (2003) GPS: Theory, Algorithms and Applications. Springer-Verlag, Berlin.

https://books.google.com/books?id=aRKPAXBt174C\&pg=PA240\&lpg=PA240\&dq= \%22general+relativity+acceleration $\% 22 \&$ source $=$ bl\&ots $=\mathrm{NnD}-\mathrm{YVx} 9 \mathrm{Go} \& \mathrm{sig}=\mathrm{ACfU}$ 3U3pvauEbW74ZuxzVIZr9n_KTb7qTw\&hl=en\&sa=X\&ved=2ahUKEwidiaDnhJHq AhVScq0KHXRBBdMQ6AEwCHoECAsQAQ\#v=onepage\&q=\%22general\%20relati vity $\% 20$ acceleration $\% 22 \& \mathrm{f}=$ false

[19] Dirac, P.A.M. (1938) A New Basis for Cosmology. Proceedings of the Royal Society $A, 165,199-208$. https://doi.org/10.1098/rspa.1938.0053

[20] Hoyle, F. and Narlikar, J.V. (1964) A New Theory of Gravitation. Proceedings of the Royal Society A, 282, 191-207. https://doi.org/10.1098/rspa.1964.0227

[21] Dirac, P.A.M. (1974) Cosmological Models and the Large Numbers Hypothesis. Proceedings of the Royal Society A, 338, 439-446.

https://doi.org/10.1098/rspa.1974.0095

[22] Boehm, C., Fayet, P. and Silk, J. (2004) Light and Heavy Dark Matter Particles. Physical Review D, 69, Article ID: 101302. https://doi.org/10.1103/PhysRevD.69.101302

[23] Arrenberg, S., et al. (2013) Complementarity of Dark Matter Experiments. http://www-public.slac.stanford.edu/snowmass2013/docs/CosmicFrontier/Comple mentarity-27.pdf

[24] Heeck, J. and Zhang, H. (2013) Exotic Charges, Multicomponent Dark Matter and Light Sterile Neutrinos. Journal of High Energy Physics, 2013, Article No. 164. https://doi.org/10.1007/JHEP05(2013)164

[25] Aoki, M., et al. (2012) Multi-Component Dark Matter Systems and Their Observa- 
tion Prospects. Physical Review D, 86, Article ID: 076015.

[26] Kusenko, A., Loewenstein, M. and Yanagida, T. (2013) Moduli Dark Matter and the Search for Its Decay Line Using Suzaku X-Ray Telescope. Physical Review D, 87, Article ID: 043508. https://doi.org/10.1103/PhysRevD.87.043508

[27] Feldman, D., Liu, Z., Nath, P. and Peim, G. (2010) Multicomponent Dark Matter in Supersymmetric Hidden Sector Extensions. Physical Review D, 81, Article ID: 095017. https://doi.org/10.1103/PhysRevD.81.095017

[28] Feng, J.L. (2010) Dark Matter Candidates from Particle Physics and Methods of Detection. Annual Review of Astronomy and Astrophysics, 48, 495-545. https://doi.org/10.1146/annurev-astro-082708-101659

[29] Zurek, K.M. (2009) Multicomponent Dark Matter. Physical Review D, 79, Article ID: 115002. https://doi.org/10.1103/PhysRevD.79.115002

[30] Spolyar, D., Freese, K. and Gondolo, P. (2007) Dark Matter and the First Stars: A New Phase of Stellar Evolution. Physical Review Letters, 100, Article ID: 051101.

[31] Ripamonti, E. and Abel, T. (2005) The Formation of Primordial Luminous Objects. arXiv: 0507130.

[32] Lee, B.W. and Weinberg, S. (1977) Cosmological Lower Bound on Heavy-Neutrino Masses. Physical Review Letters, 39, 165-168.

https://doi.org/10.1103/PhysRevLett.39.165

[33] Dicus, D.A., Kolb, E.W. and Teplitz, V.L. (1977) Cosmological Upper Bound on HeavyNeutrino Lifetimes. Physical Review Letters, 39, 168-171.

https://doi.org/10.1103/PhysRevLett.39.168

[34] Dicus, D.A., Kolb, E.W. and Teplitz, V.L. (1978) Cosmological Implications of Massive, Unstable Neutrinos. Astrophysical Journal, 221, 327-341.

https://doi.org/10.1086/156031

[35] Gunn, J.E., et al. (1978) Some Astrophysical Consequences of the Existence of a Heavy Stable Neutral Lepton. Astrophysical Journal, 223, 1015-1031.

https://doi.org/10.1086/156335

[36] Stecker, F.W. (1978) The Cosmic Gamma-Ray Background from the Annihilation of Primordial Stable Neutral Heavy Leptons. Astrophysical Journal, 223, 1032-1036. https://doi.org/10.1086/156336

[37] Zeldovich, Y.B., Klypin, A.A., Khlopov, M.Y. and Chechetkin, V.M. (1980) Astrophysical Constraints on the Mass of Heavy Stable Neutral Leptons. Soviet Journal of Nuclear Physics, 31, 664-669.

[38] Kroupa, P. (2014) Galaxies as Simple Dynamical Systems: Observational Data Disfavor Dark Matter and Stochastic Star Formation. Canadian Journal of Physics, 93, 169-202. https://doi.org/10.1139/cjp-2014-0179

[39] Netchitailo, V. (2019) Dark Matter Cosmology and Astrophysics. Journal of High Energy Physics, Gravitation and Cosmology, 5, 999-1050.

https://doi.org/10.4236/jhepgc.2019.54056

[40] Netchitailo, V.S. (2013) Word-Universe Model. https://vixra.org/abs/1303.0077

[41] Bestenlehner, J.M., et al. (2020) The R136 Star Cluster Dissected with Hubble Space Telescope/STIS. II. Physical Properties of the Most Massive Stars in R136. Monthly Notices of the Royal Astronomical Society, 499, 1918-1936.

https://doi.org/10.1093/mnras/staa2801

[42] Mehrgan, K., et al. (2019) A 40 Billion Solar Mass Black Hole in the Extreme Core of Holm 15A, the Central Galaxy of Abell 85. The Astrophysical Journal, 887, Article 
No. 195. https://doi.org/10.3847/1538-4357/ab5856

[43] Shemmer, O., et al. (2004) Near Infrared Spectroscopy of High-Redshift Active Galactic Nuclei. I. A Metallicity-Accretion Rate Relationship. The Astrophysical Journal, 614, 547-557. https://doi.org/10.1086/423607

[44] Argüelles, C.R., et al. (2021) On the Formation and Stability of Fermionic Dark Matter Haloes in a Cosmological Framework. Monthly Notices of the Royal Astronomical Society, 502, 4227-4246. https://doi.org/10.1093/mnras/staa3986

[45] Bliss, L. (2014) The Milky Way's ‘City’ Just Got a New Name. Bloomberg CityLab. https://www.bloomberg.com/news/articles/2014-09-03/the-milky-way-s-city-just-go t-a-new-name

[46] Heymans, C., et al. (2008) The Dark Matter Environment of the Abell 901/902 Supercluster: A Weak Lensing Analysis of the HST STAGES Survey. Monthly Notices of the Royal Astronomical Society, 385, 1431-1442. https://doi.org/10.1111/j.1365-2966.2008.12919.x

[47] Zwicky, F. (1933) Die Rotverschiebung von extragalaktischen Nebeln. Helvetica Physica Acta, 6, 110-127.

[48] Carr, B., Kühnel, F. and Visinelli, L. (2021) Constraints on Stupendously Large Black Holes. Monthly Notices of the Royal Astronomical Society, 501, 2029-2043. https://doi.org/10.1093/mnras/staa3651

[49] Netchitailo, V.S. (2019) Solar System. Angular Momentum. New Physics. Journal of High Energy Physics, Gravitation and Cosmology, 5, 112-139. https://doi.org/10.4236/jhepgc.2019.51005

[50] Netchitailo, V.S. (2020) World-Universe Model. Self-Consistency of Fundamental Physical Constants. https://vixra.org/abs/2006.0057

[51] Li, Q., et al. (2018) Measurements of the Gravitational Constant Using Two Independent Methods. Nature, 560, 582-588.

https://doi.org/10.1038/s41586-018-0431-5

[52] Fixsen, D.J. (2009) The Temperature of the Cosmic Microwave Background. The Astrophysical Journal, 707, 916-920. https://doi.org/10.1088/0004-637X/707/2/916

[53] Bennett, C.L., et al. (2013) Nine-Year Wilkinson Microwave Anisotropy Probe (WMAP) Observations: Final Maps and Results. Astrophysical Journal Supplement Series, 208, Article No. 20.

[54] Freedman, W.L., et al. (2020) Calibration of the Tip of the Red Giant Branch (TRGB). The Astrophysical Journal, 891, Article No. 57.

[55] Netchitailo, V.S. (2020) Hypersphere World-Universe Model: Evolution of the World. Journal of High Energy Physics, Gravitation and Cosmology, 7, 508-530. https://vixra.org/abs/2011.0209

[56] Cool Cosmos (2021) What Are Galaxies? https://coolcosmos.ipac.caltech.edu/ask/216-What-are-galaxies

[57] Ghez, A.M., et al. (2008) Measuring Distance and Properties of the Milky Way's Central Supermassive Black Hole with Stellar Orbits. The Astrophysical Journal, 689, 1044-1062. https://doi.org/10.1086/592738

[58] Lu, R., et al. (2018) Detection of Intrinsic Source Structure at $~ 3$ Schwarzschild radii with Millimeter-VLBI observations of SAGITTARIUS A*. The Astrophysical Journal, 859, Article No. 60. https://doi.org/10.3847/1538-4357/aabe2e

[59] Chou, F., Anderson, F. and Watzke, M. (2015) Release 15-001-NASA's Chandra Detects Record-Breaking Outburst from Milky Way's Black Hole. NASA.

[60] Mills, E.A.C. (2020) Journey to the Center of the Galaxy: Following the Gas to Un- 
derstand Past and Future Activity in Galaxy Nuclei. 236th Meeting of the American Astronomical Society, 1-3 June 2020, 102 p. https://aas.org/meetings/aas236 https://mills.ku.edu/files/AAS 236 wide.pdf

[61] Hooper, D. and Goodenough, L. (2011) Dark Matter Annihilation in the Galactic Center as Seen by the Fermi Gamma Ray Space Telescope. Physics Letters B, 697, 412-428. https://doi.org/10.1016/j.physletb.2011.02.029

[62] Su, M. and Finkbeiner, D.P. (2012) Evidence for Gamma-Ray Jets in the Milky Way. The Astrophysical Journal, 753, Article No. 61. https://doi.org/10.1088/0004-637X/753/1/61

[63] Ponti, G., et al. (2019) An X-Ray Chimney Extending Hundreds of Parsecs above and below the Galactic Centre. Nature, 567, 347-350. https://doi.org/10.1038/s41586-019-1009-6

[64] Bond, H.E., et al. (2013) HD 140283: A Star in the Solar Neighborhood that Formed Shortly after the Big Bang. The Astrophysical Journal Letters, 765, L12. https://doi.org/10.1088/2041-8205/765/1/L12

[65] Marchetti, T., Rossi, E.M. and Brown. A.G.A. (2018) Gaia DR2 in 6D: Searching for the Fastest Stars in the Galaxy. Monthly Notices of the Royal Astronomical Society, 490, 157-171. https://doi.org/10.1093/mnras/sty2592

[66] Koposov, S.E., et al. (2019) The Great Escape: Discovery of a Nearby $1700 \mathrm{~km} / \mathrm{s} \mathrm{Star}$ Ejected from the Milky Way by Sgr A*. Monthly Notices of the Royal Astronomical Society, 491, 2465-2480. https://doi.org/10.1093/mnras/stz3081

[67] Clarke, C.J., et al. (2018) High-Resolution Millimeter Imaging of the CI Tau Protoplanetary Disk: A Massive Ensemble of Protoplanets from 0.1 to 100 au. The Astrophysical Journal Letters, 866, L6. https://doi.org/10.3847/2041-8213/aae36b

[68] Aguilar, D.A. and Pulliam, C. (2010) Astronomers Find Giant, Previously Unseen Structure in our Galaxy. Harvard-Smithsonian Center for Astrophysics. NASA.

[69] Yang, L. and Razzaque, S. (2019) Constraints on Very High Energy Gamma-Ray Emission from the Fermi Bubbles with Future Ground-Based Experiments. Physical Review D, 99, Article ID: 083007. https://doi.org/10.1103/PhysRevD.99.083007

[70] Beall, J.H. (2015) A Review of Astrophysical Jets. Proceedings of the XI Multifrequency Behaviour of High Energy Cosmic Sources Workshop, Palermo, 25-30 May 2015, Article ID: 58.

[71] Hooper, D. and Slatyer, T.R. (2013) Two Emission Mechanisms in the Fermi Bubbles: A Possible Signal of Annihilating Dark Matter. Physics of the Dark Universe, 2, 118-138. https://doi.org/10.1016/j.dark.2013.06.003

[72] Fossat, E., et al. (2017) Asymptotic g Modes: Evidence for a Rapid Rotation of the Solar Core. arXiv: 1708.00259.

[73] Zhang, J., et al. (2005) Inner Core Differential Motion Confirmed by Earthquake Waveform Doublets. Science, 309, 1357-1360.

https://doi.org/10.1126/science.1113193

[74] Guillot, T., et al. (2018) A Suppression of Differential Rotation in Jupiter's Deep Interior. Nature, 555, 227-230. https://www.nature.com/articles/nature25775

[75] Wu, W., Ni, S. and Irving, J.C.E. (2019) Inferring Earth's Discontinuous Chemical Layering from the 660-Kilometer Boundary Topography. Science, 363, 736-740. https://doi.org/10.1126/science.aav0822

[76] Princeton University (2019) Massive Bolivian Earthquake Reveals Mountains 660 Kilometers below Our Feet. https://phys.org/news/2019-02-massive-bolivian-earthquake-reveals-mountains.ht $\underline{\mathrm{ml}}$ 
[77] Markenscoff, X. (2021) "Volume Collapse" Instabilities in Deep-Focus Earthquakes: A Shear Source Nucleated and Driven by Pressure. Journal of the Mechanics and Physics of Solids, 152, Article ID: 104379.

https://www.researchgate.net/publication/349643349 Volume collapse instabilities in deep-focus earthquakes A shear source nucleated and driven by pressure https://doi.org/10.1016/j.jmps.2021.104379

[78] Jones, G. and Bikos, K. (2020) Earth Is in a Hurry in 2020. https://www.timeanddate.com/time/earth-faster-rotation.html

[79] Margot, J.L., et al. (2021) Spin State and Moment of Inertia of Venus. Nature Astronomy, 5, 676-683. https://doi.org/10.1038/s41550-021-01339-7

[80] Cole, G.H.A. and Woolfson, M.M. (2002) Planetary Science: The Science of Planets around Stars. Taylor \& Francis, New York. https://doi.org/10.1201/9781420056853

[81] Kinver, M. (2009) Global Average Temperature May Hit Record Level in 2010. British Broadcasting Corporation, London. http://news.bbc.co.uk/2/hi/science/nature/8406839.stm

[82] Gando, A., et al. (2011) Partial Radiogenic Heat Model for Earth Revealed by Geoneutrino Measurements. Nature Geoscience, 4, 647-651. https://doi.org/10.1038/ngeo1205

[83] Elkins-Tanton, L.T. (2006) Jupiter and Saturn. Chelsea House, New York.

[84] O’Donoghue, J., Moore, L., Stallard, T.S., and Melin, H. (2016) Heating of Jupiter's Upper Atmosphere above the Great Red Spot. Nature, 536, 190-192. https://doi.org/10.1038/nature18940

[85] de Pater, I. and Lissauer, J.J. (2010) Planetary Sciences. 2nd Edition, Cambridge University Press, Cambridge. https://doi.org/10.1017/CBO9780511780561

[86] Brewster Kahle, Founder and Internet Archive (2004) Class 12-Giant PlanetsHeat and Formation. Colorado University, Boulder. https://web.archive.org/web/20080621120100/

[87] Pearl, J.C. and Conrath, B.J. (1991) The Albedo, Effective Temperature, and Energy Balance of Neptune, as Determined from Voyager Data. Journal of Geophysical Research: Space Physics, 96, 18921-18930. https://doi.org/10.1029/91JA01087

[88] Kamata, S., et al. (2019) Pluto's Ocean is Capped and Insulated by Gas Hydrates. Nature Geoscience, 12, 407-410. https://doi.org/10.1038/s41561-019-0369-8

[89] Hoffman, D.C., et al. (1971) Detection of Plutonium-244 in Nature. Nature, 234, 132-134. https://doi.org/10.1038/234132a0

[90] Wallner, A., et al. (2021) ${ }^{60} \mathrm{Fe}$ and ${ }^{244} \mathrm{Pu}$ Deposited on Earth Constrain the r-Process Yields of Recent Nearby Supernovae. Science, 372, 742-745. https://doi.org/10.1126/science.aax3972

[91] Ricard, Y. (2009) 2. Physics of Mantle Convection. In: Bercovici, D., Ed., Treatise on Geophysics. Mantle Dynamics. Elsevier, Amsterdam.

[92] Amenomori, M., et al. (2021) First Detection of Sub-PeV Diffuse Gamma Rays from the Galactic Disk: Evidence for Ubiquitous Galactic Cosmic Rays beyond PeV Energies. arXiv: 2104.05181.

[93] Gough, D.O. (1981) Solar Interior Structure and Luminosity Variations. Solar Physics, 74, 21-34. https://doi.org/10.1007/BF00151270

[94] Peter, H. (2004) Structure and Dynamics of the Low Corona of the Sun. Reviews in Modern Astronomy, 17, Article No. 87.

[95] Abhyankar, K.D. (1977) A Survey of the Solar Atmospheric Models. Bulletin of the 
Astronomical Society of India, 5, 40-44.

[96] Fox, K.C. (2014) NASA's STEREO Maps Much Larger Solar Atmosphere than Previously Observed.

https://www.nasa.gov/content/goddard/nasas-stereo-maps-much-larger-solar-atmo sphere-than-previously-observed/

[97] Aschwanden, M.J. (2004) Physics of the Solar Corona. An Introduction. Praxis Publishing, Moscow.

[98] Schmelz, J.T., et al. (2012) Composition of the Solar Corona, Solar Wind, and Solar Energetic Particles. The Astrophysical Journal, 755, Article No. 33.

http://iopscience.iop.org/article/10.1088/0004-637X/755/1/33/pdf https://doi.org/10.1088/0004-637X/755/1/33

[99] Grossman, L. (2018) Strange Gamma Rays from the Sun May Help Decipher Its Magnetic Fields.

https://www.sciencenews.org/article/strange-gamma-rays-sun-magnetic-fields

[100] Baliukin, I.I., et al. (2019) SWAN/SOHO Lyman- $\alpha$ Mapping: The Hydrogen Geocorona Extends Well beyond the Moon. Journal of Geophysical Research: Space Physics, 124, 861-885. https://doi.org/10.1029/2018JA026136

[101] Reyes, R. (2012) Exploring the Sun-Earth Connection. Southwest Research Institute. https://www.nasa.gov/sites/default/files/atoms/files/o53788232.pdf

[102] Kameda, S., et al. (2017) Ecliptic North-South Symmetry of Hydrogen Geocorona. Geophysical Research Letter, 44, 11706-11712.

https://doi.org/10.1002/2017GL075915

[103] Kuwabara, M., et al. (2017) The Geocoronal Responses to the Geomagnetic Disturbances. Journal of Geophysical Research: Space Physics, 122, 1269-1276.

https://agupubs.onlinelibrary.wiley.com/doi/pdf/10.1002/2016JA023247 https://doi.org/10.1002/2016JA023247

[104] NASA (2012) Solar System. http://chandra.harvard.edu/xray sources/solar system.html

[105] Wargelin, B.J., et al. (2014) Observation and Modeling of Geocoronal Charge Exchange X-Ray Emission During Solar Wind Gusts. The Astrophysical Journal, 796, Article No. 28. https://doi.org/10.1088/0004-637X/796/1/28

[106] Cravensa, T.E., et al. (2009) Solar Wind Charge Exchange Contributions to the Diffuse X-Ray Emission. AIP Conference Proceedings, 1156, Article No. 37. https://doi.org/10.1063/1.3211832

[107] Netchitailo, V. (2015) 5D World-Universe Model Space-Time-Energy. Journal of High Energy Physics, Gravitation and Cosmology, 1, 25-34. https://doi.org/10.4236/jhepgc.2015.11003

[108] Netchitailo, V. (2020) Hypersphere World-Universe Model: Basic Ideas. Journal of High Energy Physics, Gravitation and Cosmology, 6, 710-752. https://doi.org/10.4236/jhepgc.2020.64049 